\title{
New multiple hyper-regulus planes
}

\author{
Norman L. Johnson
}

go back

\section{Abstract}

New classes of multiple hyper-regulus translation planes of orders $q^{n}$, for $n \geq 3$, are constructed that extend the classes of Culbert-Ebert planes of orders $q^{3}$.

MSC 2000: primary: 51E23; secondary: 51A40

Keywords : reguli, hyper-reguli, multiple hyper-regulus planes

\section{Introduction}

This article considers the possibility of the construction of translation planes of order $q^{n}$ and kernel containing $\operatorname{GF}(q)$ by the replacement of a set of mutually disjoint hyper-reguli. A 'hyper-regulus' is a vector space partial spread $\mathcal{H}$ of degree $\left(q^{n}-1\right) /(q-1)$ and order $q^{n}$ which has a replacement 'hyper-regulus' spread $\mathcal{H}^{*}$, each of whose components intersects each of the components of $\mathcal{H}$, which is to say that the intersections between the two hyper-reguli are 1dimensional GF $(q)$-subspaces. Hence, when $n=2$, a hyper-regulus is a regulus.

Consider a Desarguesian plane $\Sigma$ with spread

$$
x=0, y=x m ; m \in \mathrm{GF}\left(q^{n}\right) .
$$

Define the partial spread

$$
A_{\delta}=\left\{y=x m ; m^{\left(q^{n}-1\right) /(q-1)}=\delta ; m \in \mathrm{GF}\left(q^{n}\right)^{*}\right\} .
$$

Then there are replacement partial spreads

$$
A_{\delta}^{i}=\left\{y=x^{q^{i}} m ; m^{\left(q^{n}-1\right) /(q-1)}=\delta ; m \in \mathrm{GF}\left(q^{n}\right)^{*}\right\}
$$

and since for $(i, n)=1$, the intersections between the two partial spreads are 1-dimensional GF $(q)$-subspaces, we obtain a pair of hyper-reguli. Such hyperreguli are called 'André hyper-reguli'. 




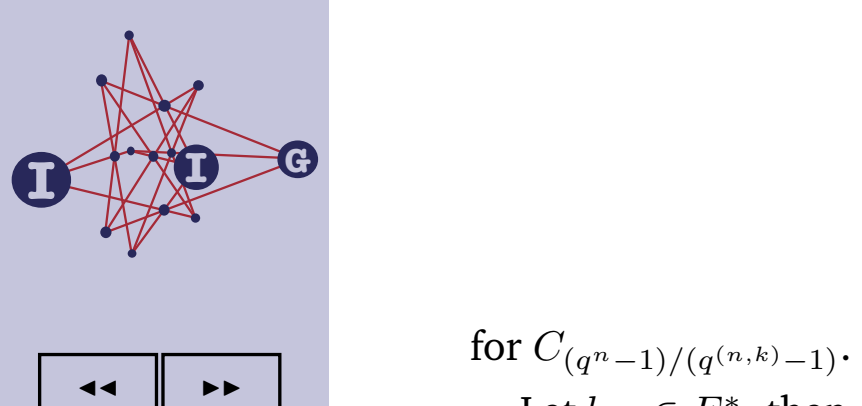

Let $b, c \in F_{q^{n}}^{*}$ then $(b, c)$ is said to be an ' $(e, \omega, \lambda)$-group admissible pair' if and only if

$$
\left(\frac{b}{c}\right)^{\left(q^{n}-1\right) /\left(q^{(n, k)}-1\right)} \notin C_{\left(q^{(n, k)}-1\right) / e} .
$$

Theorem 2.5 (Jha and Johnson [5]). Assume that $(b, c)$ is an '(e, $\omega, \lambda)$-group admissible pair' and let $\mathcal{C}_{\omega, f}$ be a coset representative set of $C_{\left(q^{n}-1\right) /\left(q^{(n, k)}-1\right)}$ as in Definition 2.4. Let

$$
\mathcal{H}_{e,(n, k)}^{*}=\left\{\begin{array}{c}
y=x^{q^{k}} \omega^{i_{j}} f\left(i_{j}\right) c d^{1-q^{k}}+x^{q^{n-k}} \omega^{-i_{j} q^{n-k}} f\left(i_{j}\right)^{-q^{n-k}} b d^{1-q^{n-k}} ; \\
d \in \mathrm{GF}\left(q^{n}\right)^{*}, \text { for } i_{j} \in \lambda
\end{array}\right\} .
$$

Then $\mathcal{H}_{e,(n, k)}^{*}$ is a set of $\left(q^{(n, k)}-1\right) / e$ mutually disjoint hyper-reguli of order $q^{n}$ and degree $\left(q^{n}-1\right) /\left(q^{(n, k)}-1\right)$.

\subsection{Non-group constructions}

If $q$ is odd, we may obtain sets of $\left(q^{(n, k)}-1\right) / 2$ mutually disjoint hyper-reguli using $C_{\left(q^{(n, k)}-1\right) / 2}$. However, other non-group-like sets are possible.

Theorem 2.6 (Jha and Johnson [5]). If $q$ is odd, let $\lambda_{\text {odd }}=\left\{1,2, \ldots,\left(q^{(n, k)}-\right.\right.$ $3) / 2\}$ and if $q$ is even let $\lambda_{\text {even }}=\left\{1,2, \ldots,\left(q^{(n, k)} / 2-1\right\}\right.$. For either case odd or even, let $(b, c)$ be a $(\omega, \lambda)$-admissible pair for $\lambda=\lambda_{\text {odd }}$ or $\lambda_{\text {even, }}$, respectively. Let $\mathcal{C}_{\omega, f}$ be a coset representative set of $C_{\left(q^{n}-1\right) /\left(q^{(n, k)}-1\right)}$ as in Definition 2.1.

(1) If $q$ is odd let

$$
\mathcal{H}_{\left(q^{(n, k)}-3\right) / 2}^{*}=\left\{\begin{array}{c}
y=x^{q^{k}} \omega^{i_{j}} f\left(i_{j}\right) c d^{1-q^{k}}+x^{q^{n-k}} \omega^{-i_{j} q^{n-k}} f\left(i_{j}\right)^{-q^{n-k}} b d^{1-q^{n-k}} ; \\
d \in \mathrm{GF}\left(q^{n}\right)^{*}, i_{j} \in \lambda_{o d d}
\end{array}\right\} .
$$

Then $\mathcal{H}_{\left(q^{(n, k)}-1\right) / 3}^{*}$ is a set of $\left(q^{(n, k)}-3\right) / 2$ mutually disjoint hyper-reguli of order $q^{n}$ and degree $\left(q^{n}-1\right) /\left(q^{(n, k)}-1\right)$.

(2) If $q$ is even let

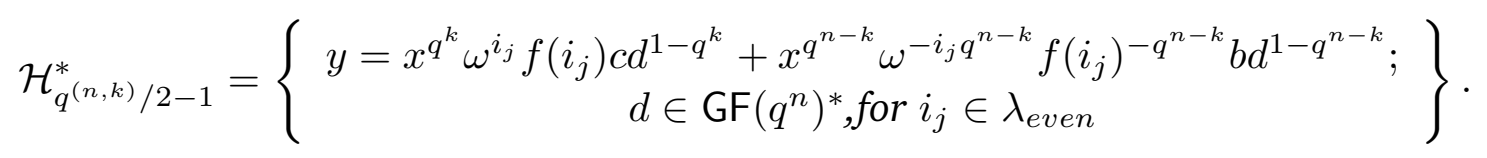

Then $\mathcal{H}_{q^{(n, k)} / 2-1}^{*}$ is a set of $q^{(n, k)} / 2-1$ mutually disjoint hyper-reguli of order $q^{n}$ and degree $\left(q^{n}-1\right) /\left(q^{(n, k)}-1\right)$.

Remark 2.7. If a choice of subset $\lambda$ produces a set of mutually disjoint hyperreguli, so does $\lambda+i_{0}$, for any fixed integer $i_{0}$, for $i_{0}=1,2, \ldots,\left(q^{(n, k)}-1\right)$. 


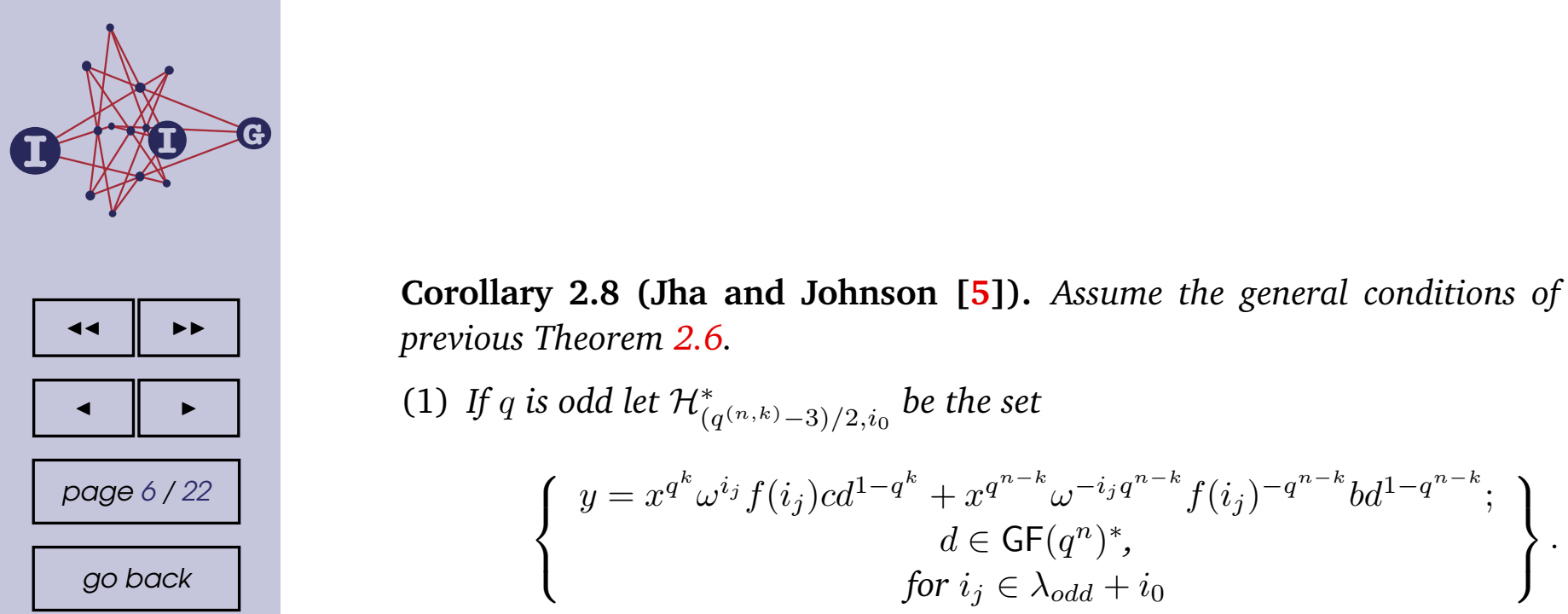

full screen

close

Then $\mathcal{H}_{\left(q^{(n, k)}-3\right) / 2, i_{0}}^{*}$ is a set of $\left(q^{(n, k)}-3\right) / 2$ mutually disjoint hyper-reguli of order $q^{n}$ and degree $\left(q^{n}-1\right) /\left(q^{(n, k)}-1\right)$.

(2) If $q$ is even let $\mathcal{H}_{q^{(n, k)} / 2-1, i_{0}}^{*}$ be the set

$$
\left\{\begin{array}{c}
y=x^{q^{k}} \omega^{i_{j}} f\left(i_{j}\right) c d^{1-q^{k}}++x^{q^{n-k}} \omega^{-i_{j} q^{n-k}} f\left(i_{j}\right)^{-q^{n-k}} b d^{1-q^{n-k}} \\
d \in \mathrm{GF}\left(q^{n}\right)^{*} \\
\text { for } i_{j} \in \lambda_{\text {even }}+i_{0}
\end{array}\right\} .
$$

Then $\mathcal{H}_{q^{(n, k)} / 2-1, i_{0}}^{*}$ is a set of $q^{(n, k)} / 2-1$ mutually disjoint hyper-reguli of order $q^{n}$ and degree $\left(q^{n}-1\right) /\left(q^{(n, k)}-1\right)$.

\section{Culbert-Ebert 'Sherk surfaces'}

Part of the following section also appears in the 'Handbook of Finite Translation Planes' by N.L. Johnson, V. Jha and M. Biliotti, Taylor Books, 2007, and ultimately depends on the work of Culbert-Ebert. It is repeated here for convenience of exposition.

Culbert and Ebert [3] have constructed various sets of hyper-reguli of order $q^{3}$ and degree $1+q+q^{2}$. Bruck [1] shows that any hyper-regulus in a Desarguesian affine plane of degree $1+q+q^{2}$ and order $q^{3}$ is actually an André hyper-regulus and Pomareda [8] showed there are actually two possible replacements, namely the André replacements. Recall that in the standard setting there are $q-1$ mutually disjoint André hyper-regulus with two components $x=0, y=0$ and a corresponding affine homology group $H$ of order $1+q+q^{2}$ leaving invariant each André hyper-regulus. A subset of hyper-regular is said to be 'linear' if and only if the set is a subset of an André set of $q-1$ hyper-reguli which are orbits under a group isomorphic to $H$.

Culbert and Ebert [3] actually found sets of mutually disjoint hyper-reguli (so each is necessarily André) with the property that no subset of at least two hyperreguli is linear. Any such subset then can be replaced in two ways producing a translation plane which cannot be André or indeed generalized André. 





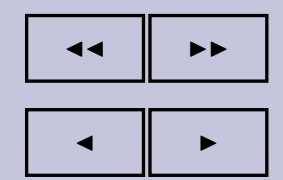

page $10 / 22$

go back

full screen

close

quit

for $u$ a fixed non-zero element of $\operatorname{GF}(q)$, where $q$ is even. Clearly, $S(1,0, u, 0)$ and $S(0,0,0,1)$ are disjoint sets. Within this set is the subset

$$
\left\{S\left(1,0, u, \frac{u^{2}}{d}+u d\right) ; \text { for } u \neq d^{2} \text { in } \operatorname{GF}(q)^{*}\right\} .
$$

According to the criteria established for hyper-reguli 'covers' for even order, we recall part (3) of Theorem 3.3. Let $S=S(1, \alpha, \delta, g)$ be a Sherk surface that is not a single point. Define $\delta^{\prime}=\delta+\alpha^{q^{2}+q}$ and $g^{\prime}=g+T(\alpha \delta)$. Then $S$ is a cover if $g \neq T(\alpha \delta)$ and $T_{0}\left(c^{\prime}\right)=0$ where $c^{\prime}=N\left(\delta^{\prime}\right) /\left(g^{\prime}\right)^{2}$. Since $\alpha=0$ and $\delta=u=\delta^{\prime} m$ we see that $g^{\prime}=g=\frac{u^{2}}{d}+u d$ and $c^{\prime}=u^{3} /\left(\frac{u^{2}}{d}+u d\right)^{2}$.

We claim that

$$
T_{0}\left(u^{3} /\left(\frac{u^{2}}{d}+u d\right)^{2}\right)=0
$$

for all such elements $d$ in $\operatorname{GF}(q)^{*}$, such that $u \neq d^{2}$. We note that $u^{3} /\left(\frac{u^{2}}{d}+u d\right)^{2}=$ $d^{2} u /\left(u^{2}+d^{4}\right)$. Let $q=2^{r}$, and recall that $T_{0}(t)=T_{0}\left(t^{2}\right)$. Hence,

$$
T_{0}\left(u^{3} /\left(\frac{u^{2}}{d}+u d\right)^{2}\right)=T_{0}\left(d u^{2^{r-1}} /\left(u+d^{2}\right)\right) .
$$

Now let

$$
\begin{aligned}
d u^{2^{r-1}} /\left(u+d^{2}\right) & =d\left(u+d^{2}+d^{2}\right)^{2^{r-1}} /\left(u+d^{2}\right) \\
& =d\left(u+d^{2}\right)^{2^{r-1}-1}+d d^{2^{2^{r-1}}} /\left(u+d^{2}\right) \\
& =d\left(u+d^{2}\right)^{2^{r-1}-1}+d^{2}\left(u+d^{2}\right)^{-1}
\end{aligned}
$$

Furthermore, notice that

$$
d^{2}\left(u+d^{2}\right)^{-1}=d^{2}\left(u+d^{2}\right)^{q-2}=\left(\left(d\left(u+d^{2}\right)\right)^{2^{r-1}-1}\right)^{2} .
$$

Also,

$$
T_{0}\left(\left(\left(d\left(u+d^{2}\right)\right)^{2^{r-1}-1}\right)^{2}\right)=T_{0}\left(\left(d\left(u+d^{2}\right)\right)^{2^{r-1}-1}\right) .
$$

Thus,

$$
\begin{aligned}
T_{0}\left(u^{3} /\left(\frac{u^{2}}{d}+u d\right)^{2}\right) & =T_{0}\left(d u^{2^{r-1}} /\left(u+d^{2}\right)\right) \\
& =T_{0}\left(d\left(u+d^{2}\right)^{2^{r-1}-1}+d^{2}\left(u+d^{2}\right)^{-1}\right) \\
& =T_{0}\left(d\left(u+d^{2}\right)^{2^{r-1}-1}\right)+T_{0}\left(\left(d\left(u+d^{2}\right)^{2^{r-1}-1}\right)^{2}\right) \\
& =T_{0}\left(d\left(u+d^{2}\right)^{2^{r-1}-1}\right)+T_{0}\left(d\left(u+d^{2}\right)^{2^{r-1}-1}\right)=0 .
\end{aligned}
$$

Hence, we have a set of mutually disjoint hyper-reguli. Note that

$$
\frac{u^{2}}{d}+u d=\frac{u^{2}}{e}+u e
$$





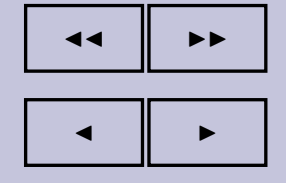

page $12 / 22$

go back

full screen

close

quit which produces the following equation:

$$
\begin{aligned}
(f-1) N(x)+T\left((a-f b)^{q^{2}}\right) & \left.x^{1+q}\right) \\
& +T\left(\left(f b^{q+q^{2}}-a^{q+q^{2}}\right) x\right)+N(a)-f N(b)=0,
\end{aligned}
$$

which determines the Sherk surface $S\left(f-1, a-f b, f b^{q+q^{2}}-a^{q+q^{2}}, N(a)-\right.$ $f N(b))$. Hence, we obtain:

Lemma 5.1. The hyper-regulus

$$
N\left(\frac{x-a}{x-b}\right)=f
$$

for $x \neq b$ is given by the Sherk surface

$$
S\left(f-1, a-f b, f b^{q+q^{2}}-a^{q+q^{2}}, N(a)-f N(b)\right) .
$$

We now apply this lemma to the two Sherk pencils of Culbert and Ebert of Theorem 3.4(i), as well as to the new Sherk pencil of the previous section.

Case (i). Let $q$ be an odd prime power $\geq 7$.

Consider the $F$-linear combination

$$
f S(1,0,-1,0)+g S(0,0,0,1) ; f, g \in \mathrm{GF}(q) .
$$

Then the subset of (mutually disjoint) covers has cardinality $\frac{(q-3)}{2}$. Furthermore, this set is

$$
\left\{\left(S(1,0,-1, g) ;-4+g^{2} \text { is a non-zero square in } \operatorname{GF}(q), g \in \mathrm{GF}(q)\right\} .\right.
$$

Case (ii). Let $q$ be an odd prime power $\geq 5$.

Let $u$ be a fixed non-square. Consider the $F$-linear combination

$$
f S(1,0,-u, 0)+g S(0,0,0,1) ; f, g \in \mathrm{GF}(q) .
$$

Then the subset of (mutually disjoint) covers has cardinality $\frac{(q-1)}{2}$. This subset of covers is

$$
\left\{S(1,0,-u, g) ;-4 u^{3}+g^{2} \text { a non-zero square in } \operatorname{GF}(q)\right\} .
$$




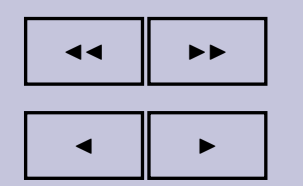

page $13 / 22$

go back

full screen

close

quit

Case (iii). Let $q$ be even.

In the previous sections, we have considered the set of $(q-2) / 2$ hyper-reguli

$$
\left\{S\left(1,0, u, \frac{u^{2}}{d}+u d\right) ; \text { for } u \neq d^{2} \text { in } \mathrm{GF}(q)^{*}, q \text { even }\right\} \text {. }
$$

We now therefore see that all of these three of these sets in cases (i), (ii), (iii) have the basic Sherk surface $S(1,0, u, g)$, for $u$ a fixed element of $\operatorname{GF}(q)^{*}$.

From Lemma 5.1, we match this with

$$
S\left(f-1,(a-f b) /(f-1),\left(f b^{q+q^{2}}-a^{q+q^{2}}\right) /(f-1),(N(a)-f N(b)) /(f-1)\right) .
$$

Therefore, $a=f b$. Now assume that $a$ and $b$ are in $\operatorname{GF}(q)$. Hence, we must have

$$
\left(f b^{q+q^{2}}-a^{q+q^{2}}\right) /(f-1)=\left(f b^{2}-a^{2}\right) /(f-1)=\left(f b^{2}-f^{2} b^{2}\right) /(f-1)=-f b^{2}=u .
$$

So,

$$
f=-u / b^{2}
$$

So, $a=-u / b$. So

$$
(N(a)-f N(b)) /(f-1))=\frac{-u^{3} / b^{3}+\left(u / b^{2}\right) b^{3}}{-\left(u / b^{2}+1\right)} .
$$

Note that $\frac{-u^{3} / b^{3}+\left(u / b^{2}\right) b^{3}}{-\left(u / b^{2}+1\right)}=\frac{-u^{3}+u b^{4}}{-b\left(u+b^{2}\right)}=\frac{u\left(b^{4}-u^{2}\right)}{-b\left(u+b^{2}\right)}=\frac{u\left(b^{2}-u\right)}{-b}=\frac{u^{2}}{b}-u b$. Thus, we have

Lemma 5.2. The hyper-regulus

$$
N\left(\frac{x-(-u / b)}{x-b}\right)=-u / b^{2}
$$

for $x \neq b$ is given by the Sherk surface

$$
S\left(1,0, u, \frac{u^{2}}{b}-u b\right)
$$

for $b^{2} \neq u$.

Theorem 5.3. The set of Sherk surfaces

$$
\left\{S\left(1,0, u, \frac{u^{2}}{d}-u d\right) ; \text { for } u \neq d^{2} \text { in } \mathrm{GF}(q)^{*}\right\}
$$

is a set of mutually disjoint hyper-reguli.

(i) When $q$ is odd and $u$ is a non-square, the set has $(q-1) / 2$ hyper-reguli. 



Note that

$$
\begin{aligned}
\left(\left(z^{*} e\right)^{-q^{-1}}-(z d)^{-q^{-1}}\right)^{\left(q^{n}-1\right) /(q-1)} & =\left(z d-z^{*} e\right)^{\left(q^{n}-1\right) /(q-1)} /\left(z d z^{*} e\right)^{\left(q^{n}-1\right) /(q-1)} \\
& =\left(z d-z^{*} e\right)^{\left(q^{n}-1\right) /(q-1)} /\left(u^{(n-1) / 2} d u^{(n-1) / 2} e\right) \\
& =\left(z d-z^{*} e\right)^{\left(q^{n}-1\right) /(q-1)} /\left(u^{n-1} d e\right) .
\end{aligned}
$$

Assume that

$$
z d=z^{*} e .
$$

Then it follows that $u^{(n-1) / 2} d=u^{(n-1) / 2} e$ so that $d=e$ and hence $z=z^{*}$, implying that the two functions are identical. Therefore, we must have that

$$
\left(z d-z^{*} e\right)^{\left(q^{n}-1\right) /(q-1)}=\left(\left(z^{*} e\right)^{-q^{-1}}-(z d)^{-q^{-1}}\right)^{\left(q^{n}-1\right) /\left(q^{n}-1\right)} u^{n}
$$

implies that

$$
d e=u .
$$

Now again using Theorem 2.3, we see, considering the two elements of $\{d, u / d\}$, that each defines a replacement hyper-regulus of the other.

Now consider the situations:

(i) $q$ odd and $u$ non-square in $\operatorname{GF}(q)$,

(ii) $q$ odd and $u$ non-zero square in $\operatorname{GF}(q)$, and

(iii) $q$ even and $u$ non-zero in $\operatorname{GF}(q)$.

Applying the argument of Theorem 5.3, we see that we obtain $(q-1) / 2$ mutually disjoint hyper-reguli in case (i), $(q-3) / 2$ mutually disjoint hyperreguli in case (ii) and $(q-2) / 2$ mutually disjoint hyper-reguli in case (iii).

Hence, we obtain the following theorem:

Theorem 6.1. Let $\Sigma$ be a Desarguesian affine plane of order $q^{n}$ with spread

$$
x=0, y=x m ; m \in \mathrm{GF}\left(q^{n}\right) .
$$

Choose any element $u$ of $\operatorname{GF}(q)^{*}$. Let $\lambda$ be any set of elements of $\operatorname{GF}(q)^{*}$ with the property that for $d, e$ in $\lambda$ then $d e \neq u$. Then

$$
\left\{\left\{y=x^{q}(z d)+x^{q^{-1}}(z d)^{-q^{-1}} u ; z^{\left(q^{n}-1\right) /(q-1)}=-u^{(n-1) / 2} / d^{n-1}\right\} ; d \in \lambda\right\}
$$

forms a set of mutually disjoint hyper-reguli.

(i) If $q$ is odd and $u$ is a non-square then $\lambda$ has cardinality $(q-1) / 2$.

(ii) If $q$ is odd and $u$ is a non-zero square that $\lambda$ has cardinality $(q-3) / 2$.

(iii) If $q$ is even and and $u$ is a non-zero element of $\mathrm{GF}(q)$ then $\lambda$ has cardinality $(q-2) / 2$. 



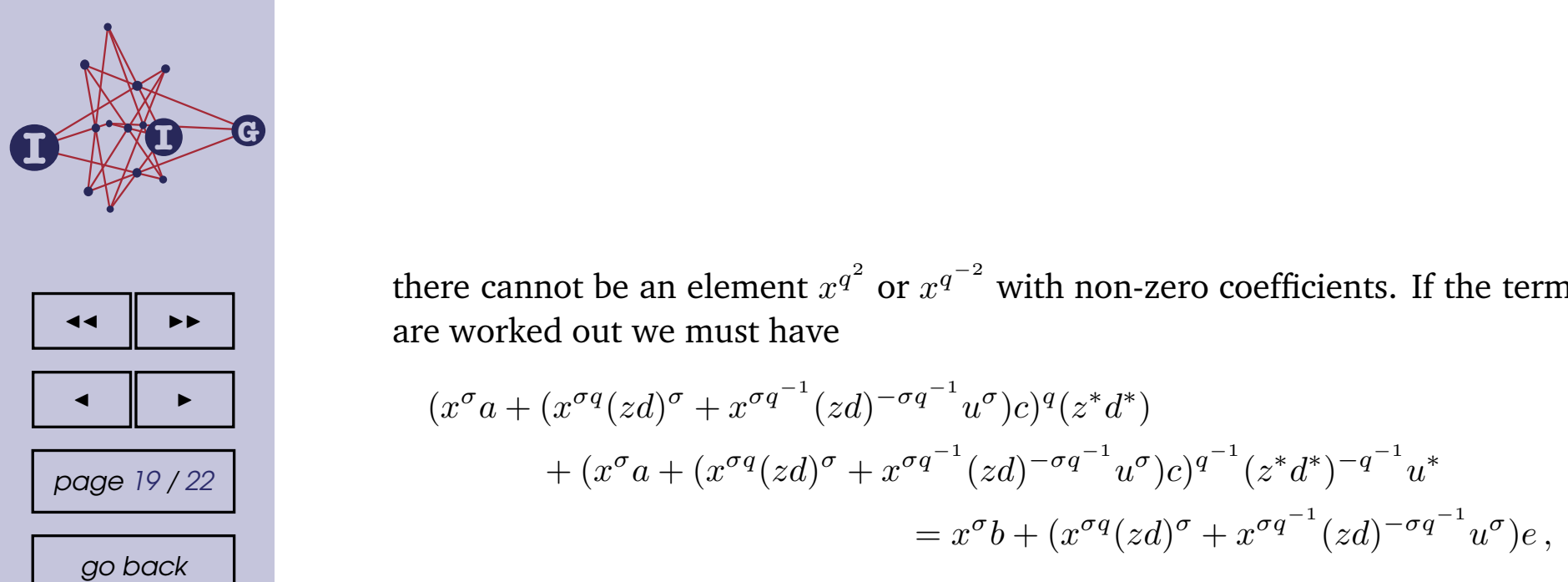

for all elements $x \in \operatorname{GF}\left(q^{n}\right)$. Since this is a polynomial identity, we see that the coefficients on the $x^{q^{2}}$ and $x^{q^{-2}}$, are both zero, since $n>3$ and hence $>4$. The coefficient on the $x^{q^{2}}$-term is $(z d)^{\sigma q} c^{q}\left(z^{*} d^{*}\right)$, forcing $c=0$. But this implies in turn that $b=0$ since this is the only coefficient on the $x$-term. Since all of the translation planes constructed admit the kernel homology group as a collineation group leaving invariant each hyper-regulus in question, it follows that we may assume that $a=1$. Hence, we obtain

$$
x^{\sigma q}\left(z^{*} d^{*}\right)+x^{\sigma q^{-1}}\left(z^{*} d^{*}\right)^{-q^{-1}} u^{*}=\left(x^{\sigma q}(z d)^{\sigma}+x^{\sigma q^{-1}}(z d)^{-\sigma q^{-1}} u^{\sigma}\right) e,
$$

for all elements $x \in \operatorname{GF}\left(q^{n}\right)$. Therefore,

$$
(z d)^{\sigma} e=z^{*} d^{*},(z d)^{-\sigma q^{-1}} u^{\sigma} e=\left(z^{*} d^{*}\right)^{-q^{-1}} u^{*} .
$$

So, we must have

$$
\left(\left(z^{*} d^{*}\right) e^{-1}\right)^{-q^{-1}}=(z d)^{\sigma-q^{-1}}=\left(z^{*} d^{*}\right)^{-q^{-1}} u^{*}\left(u^{\sigma} e\right)^{-1} .
$$

Hence, we see that

$$
e^{q+1}=u^{*}\left(u^{\sigma}\right)^{-1} .
$$

Note that since $n$ is odd, then $e^{\left(q^{2}-1\right)}=1$ and $\left(q^{2}-1, q^{n}-1\right)=q^{(2, n)}-1=q-1$. So the order of $e$ divides $q-1$. Then $e^{q+1}=e^{q-1+2}=e^{2}$ so that $e^{2}$ is in $\operatorname{GF}(q)$, implying that $\{e \alpha+\beta ; \alpha, \beta \in \mathrm{GF}(q)\}$ is a subfield of order $q^{2}$ or $q$. Since $n$ is odd, then the subfield is of order $q$, so that $e$ is in $\operatorname{GF}(q)$. Therefore, we have

$$
u^{*}=u^{\sigma} e^{2} \text { and } \lambda^{*}=\lambda^{\sigma} e .
$$

So, for example, assume that $q$ is an odd prime. Since there are $2^{|\lambda|}$ possible sets $\lambda$ for the same element $u$, we see that $e^{2}= \pm 1$, implying that there are exactly $2^{|\lambda|-1}$, mutually non-isomorphic translation planes constructed and more generally for $q=p^{r}$, there are at least $2^{|\lambda|-1} / r$. Thus, we have the following theorem.

Theorem 7.1. The translation planes $\Sigma_{\lambda, u}$ and $\Sigma_{\lambda^{*}, u^{*}}$ of order $q^{n}=p^{2 r}, p a$ prime for $n$ odd and $n>3$ are isomorphic if and only if

$$
u^{*}=u^{\sigma} e^{2} \text { and } \lambda^{*}=\lambda^{\sigma} e,
$$

where $e$ is an element of $\mathrm{GF}(q)^{*}$. Hence, there are at least $2^{|\lambda|-1} / r$ mutually disjoint translation planes. 



\section{Connecting ideas}

The new classes of translation planes that we have constructed may be connected to the main construction theorem of Jha and Johnson 2.5. Just from the general form of this result, it would appear that all of our translation planes may be placed within the context of this theorem. Still, however, such classes should be considered new examples of admissible pairs producing planes under the general theory.

To illustrate, consider the planes $\Sigma_{\lambda, u}$ in Theorem 6.1, when $q$ is odd and $u$ is a non-square. We connect this class to the class of Jha and Johnson in Theorem 2.5 , where the idea is to use the cyclic group of order $(q-1) / 2, C_{(q-1) / 2}$ of $\operatorname{GF}(q)^{*}$ and a choice of element $b$ such that $b^{\left(q^{n}-1\right) /(q-1)} \notin C_{(q-1) / 2}$. To connect these two classes of planes, recall that for $\Sigma_{\lambda, u}$, there is an implicit partition of $\mathrm{GF}(q)^{*}$ in $(q-1) / 2$ pairs $\{\beta, u / \beta\}$ to one may construct sets $\lambda$ by choosing one element out of each pair of the partition. We have pointed out that this amounts to making different choices of the at least two possible replacement hyper-reguli when $n$ is odd. Note also that if $\beta$ is a square or non-square, respectively as $u / \beta$ is non-square or square. Since $C_{(q-1) / 2}$ contains the full set of $(q-1) / 2$ nonzero squares on $\operatorname{GF}(q)$, we see that if we choose a set $\lambda$ by selecting all of the squares from the sets $\{\beta, u / \beta\}$, and choose $b=u$, the plane $\Sigma_{\lambda, u}$ and the groupconstructed plane of Jha-Johnson are identical.

We note that since general theory of Jha-Johnson is valid for arbitrary $n$, odd or even, the possible connection between admissible sets and how two such sets might be related via different choices of replacements per hyper-regulus is not considered.

\section{References}

[1] R.H. Bruck, Circle geometry in higher dimensions, A Survey of Combinatorial Theory (Proc. Internat. Sympos., Colorado State Univ., Fort Collins, Colo., 1971), North-Holland, Amsterdam, 1973, pp. 69-77.

[2] R.H. Bruck, Circle geometry in higher dimensions, II, Geom. Dedicata 2 (1973), 133-188.

[3] C. Culbert and G.L. Ebert, Circle geometry and three-dimensional subregular translation planes, Innov. Incidence Geom. 1 (2005), 3-18.

[4] V. Jha and N.L. Johnson, A new class of translation planes constructed by hyper-regulus replacement, J. Geom. (to appear). 

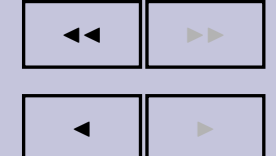

page 22 / 22

go back

full screen

close

quit
[5] $\longrightarrow$ ment, J. Geom. (to appear).

[6] Collineation groups of translation planes constructed by multiple hyper-regulus replacement, Note Mat. 26 (2006), no. 1, 149-167.

[7] _ Cubic order translation planes constructed by multiple hyper-regulus replacement, Note Mat. 26 (2006), no. 1, 79-103.

[8] R. Pomareda, Hyper-reguli in projective space of dimension 5, Mostly Finite Geometries (Iowa City, IA, 1996), Lecture Notes in Pure and Appl. Math., vol. 190, Dekker, New York, 1997, pp. 379-381.

\author{
Norman L. Johnson \\ e-mail: njohnson@math.uiowa.edu \\ Department of MATHEMATics, University of IowA, IOWA City, IOWA 52242, USA
}

